EPJ manuscript No.

(will be inserted by the editor)

\title{
Open heavy-flavour production in ALICE
}

\author{
A. Dainese for the ALICE Collaboration \\ Dipartimento di Fisica "G. Galilei”, Università degli Studi di Padova, and INFN, Italy
}

Received: November 19, 2018

\begin{abstract}
After a short review of the Physics motivations for the study of open heavy flavour production in proton-proton, proton-nucleus and nucleus-nucleus collisions at the LHC, we present results on the expected performance of the ALICE experiment for charm and beauty production measurements.
\end{abstract}

PACS. 2 5.75.-q, 14.65.Dw, 13.25.Ft

\section{Introduction}

The ALICE experiment [1] will study nucleus-nucleus (AA) collisions at the LHC, with a centre-of-mass energy $\sqrt{s_{\mathrm{NN}}}=$ 5.5 TeV per nucleon-nucleon (NN) collision for the $\mathrm{Pb}-\mathrm{Pb}$ system, in order to investigate the properties of QCD matter at energy densities of up to several hundred times the density of atomic nuclei. Under these conditions a deconfined state of quarks and gluons is expected to be formed.

The measurement of open charm and open beauty production allows to investigate the mechanisms of heavyquark production, propagation and hadronization in the hot and dense medium formed in high-energy nucleusnucleus collisions. The open charm and open beauty cross sections are also needed as a reference to measure the effect of the transition to a deconfined phase on the production of quarkonia. Heavy-quark production measurements in proton-proton and proton-nucleus collisions at the LHC, besides providing the necessary baseline for the study of medium effects in nucleus-nucleus collisions, are interesting per se, as a test of QCD in a new energy domain.

\section{Heavy-flavour production from pp to AA}

Heavy-quark pairs $(\mathrm{Q} \overline{\mathrm{Q}})$ are expected to be produced in primary partonic scatterings with large virtuality $Q^{2}>$ $\left(2 m_{\mathrm{Q}}\right)^{2}$ and, thus, on small temporal and spatial scales, $\Delta t \sim \Delta r \sim 1 / Q \lesssim 0.1 \mathrm{fm}$ (for $m_{\mathrm{c}}=1.2 \mathrm{GeV}$ ). In nucleusnucleus reactions, this implies that the initial production process is not affected by the presence of the dense medium formed in the collision. Given the large virtualities, the baseline production cross sections in NN collisions can be calculated in the framework of collinear factorization and perturbative QCD (pQCD) 2. For the estimate of baseline production yields in nuclear collisions (to be used for performance studies and preparation of the analysis strategies), a scaling of the yields with the average number $\left\langle N_{\text {coll }}\right\rangle$ of inelastic $\mathrm{NN}$ collisions (binary scaling) is usually assumed:

$$
\mathrm{d}^{2} N_{\mathrm{AA}(\mathrm{pA})}^{\mathrm{Q}} / \mathrm{d} p_{\mathrm{t}} \mathrm{d} y=\left\langle N_{\text {coll }}\right\rangle \times \mathrm{d}^{2} N_{\mathrm{pp}}^{\mathrm{Q}} / \mathrm{d} p_{\mathrm{t}} \mathrm{d} y .
$$

The expected $c \bar{c}$ and $b \bar{b}$ production yields for different collision systems at the LHC are reported in the first line of Table 13 . These numbers, assumed as the ALICE baseline, are obtained from pQCD calculations at NLO 2, including the nuclear modification of the parton distribution functions (PDFs) 4] in the $\mathrm{Pb}$ nucleus (details on the choice of $\mathrm{pQCD}$ parameter values and PDF sets can be found in 3 ). Note that the predicted yields have large uncertainties, of about a factor 2, estimated by varying the values of the calculation parameters. An illustration of the theoretical uncertainty band for the D meson cross section as a function of $p_{\mathrm{t}}$ will be shown in section 3 along with the expected sensitivity of the ALICE experiment.

Several effects can determine the breakdown of binary scaling in pA and AA collisions. They are usually divided in two classes, that we discuss in the following.

Initial-state effects, such as nuclear shadowing, the modification of the parton distribution functions in the nucleus due to gluon recombination at small momentum fraction $x$. Initial-state effects can, at least in principle, be studied by comparing proton-proton and proton-nucleus collisions. It has recently been argued that, indeed, at LHC

Table 1. Expected $\mathrm{Q} \overline{\mathrm{Q}}$ yields per event at the LHC, from NLO pQCD calculations [3]. For $\mathrm{p}-\mathrm{Pb}$ and $\mathrm{Pb}-\mathrm{Pb}, \mathrm{PDF}$ nuclear modification is included and $N_{\text {coll }}$ scaling is assumed.

\begin{tabular}{cccc}
\hline colliding system & $\mathrm{pp}$ & $\mathrm{p}-\mathrm{Pb}$ & $\mathrm{Pb}-\mathrm{Pb}$ \\
$\sqrt{s_{\mathrm{NN}}}$ & $14 \mathrm{TeV}$ & $8.8 \mathrm{TeV}$ & $5.5 \mathrm{TeV}$ \\
centrality & - & min. bias & $0-5 \% \sigma^{\text {inel }}$ \\
\hline $\mathrm{c} \overline{\mathrm{c}}$ pairs & 0.16 & 0.78 & 115 \\
$\mathrm{~b} \overline{\mathrm{b}}$ pairs & 0.0072 & 0.029 & 4.6 \\
\hline
\end{tabular}


A. Dainese for the ALICE Collaboration: Open heavy-flavour production in ALICE

energy, gluon recombination may occur even in pp collisions and affect the charm production cross section [5].

Final-state effects in AA collisions, due to the interaction of the produced partons with the medium. Partonic energy loss in the medium is the main example of such an effect. Believed to be at the origin of the jet quenching phenomena observed in $\mathrm{Au}-\mathrm{Au}$ collisions at RHIC [6], energy loss is expected to depend on the properties of the medium (gluon density and volume) and on the properties of the 'probe' parton (colour charge and mass). Due to the large values of their masses, charm and beauty quarks are qualitatively different probes with respect to light partons, since, on QCD grounds, the in-medium energy loss of massive partons is expected to be reduced relative to that of 'massless' partons (light quarks and gluons) [7,8. 9. In addition to that, since at LHC energy most of the measured light-flavour hadrons will originate from a gluon parent, heavy-flavour particles, such as D mesons, will provide a tool to tag a quark parent. As pointed out in [10, the comparison of the high- $p_{\mathrm{t}}$ suppression for $\mathrm{D}$ mesons and for light-flavour hadrons should test the colour-charge dependence (quark parent vs. gluon parent) of parton energy loss, while the comparison for B mesons and for lightflavour hadrons should test its mass dependence (massive parent vs. massless parent) — in section 4 and [5] we show some predictions from [10] and compare them to the expected ALICE sensitivity for these quenching studies.

\section{Heavy-flavour detection in ALICE}

The ALICE experimental setup 111 was designed in order to allow the detection of $\mathrm{D}$ and $\mathrm{B}$ mesons in the high-multiplicity environment of central $\mathrm{Pb}-\mathrm{Pb}$ collisions at LHC energy, where up to several thousand charged particles might be produced per unit of rapidity. The heavyflavour capability of the ALICE detector is provided by:

- Tracking system; the Inner Tracking System (ITS), the Time Projection Chamber (TPC) and the Transition Radiation Detector (TRD), embedded in a magnetic field of $0.5 \mathrm{~T}$, allow track reconstruction in the pseudorapidity range $-0.9<\eta<0.9$ with a momentum resolution better than $2 \%$ for $p_{\mathrm{t}}<20 \mathrm{GeV} / c$ and a transverse impact parameter ${ }^{1}$ resolution better than $60 \mu \mathrm{m}$ for $p_{\mathrm{t}}>1 \mathrm{GeV} / c$ (the two innermost layers of the ITS are equipped with silicon pixel detectors $)^{2}$.

- Particle identification system; charged hadrons are separated via $\mathrm{d} E / \mathrm{d} x$ in the TPC and in the ITS and via time-of-flight measurement in the Time Of Flight (TOF) detector; electrons are separated from charged pions in the dedicated Transition Radiation Detector

\footnotetext{
1 The transverse impact parameter, $d_{0}$, is defined as the distance of closest approach of the track to the interaction vertex, in the plane transverse to the beam direction.

2 Note that, for pp collisions, the impact parameter resolution maybe slightly worse, due to the larger transverse size of the beam at the ALICE interaction point. This is taken into account in the studies presented in the following.
}

(TRD) 12, and in the TPC; muons are identified in the forward muon spectrometer covering in acceptance the range $-4<\eta<-2.5$.

Detailed analyses 13 , based on a full simulation of the detector and of the background sources, have shown that ALICE has a good potential to carry out a rich heavyflavour Physics programme. In section 4 we describe the expected performance for the exclusive reconstruction of $\mathrm{D}^{0} \rightarrow \mathrm{K}^{-} \pi^{+}$decays in $\mathrm{pp}, \mathrm{p}-\mathrm{Pb}$ and $\mathrm{Pb}-\mathrm{Pb}$ collisions, and the estimated sensitivity for the study of charm energy loss in $\mathrm{Pb}-\mathrm{Pb}$ collisions. In sections [5] and 6] we present the perspectives for the measurement of beauty production in central $\mathrm{Pb}-\mathrm{Pb}$ collisions in the semi-electronic and semimuonic decay channels.

For all studies a multiplicity of $\mathrm{d} N_{\mathrm{ch}} / \mathrm{d} y=6000$ was assumed for central $\mathrm{Pb}-\mathrm{Pb}$ collisions ${ }^{3}$. We report the results corresponding to the expected statistics collected by ALICE per LHC year: $10^{7}$ central $\left(0-5 \% \sigma^{\text {inel }}\right) \mathrm{Pb}-\mathrm{Pb}$ events at $\mathcal{L}_{\mathrm{Pb}-\mathrm{Pb}}=10^{27} \mathrm{~cm}^{-2} \mathrm{~s}^{-1}$ and $10^{9}$ pp events at $\mathcal{L}_{\mathrm{pp}}^{\mathrm{ALICE}}=$ $5 \times 10^{30} \mathrm{~cm}^{-2} \mathrm{~s}^{-1}$, in the barrel detectors; the forward muon arm will collect about 40 times larger samples (i.e. $4 \times 10^{8}$ central $\mathrm{Pb}-\mathrm{Pb}$ events).

\section{Measurement of charm production and in-medium quenching}

One of the most promising channels for open charm detection is the $\mathrm{D}^{0} \rightarrow \mathrm{K}^{-} \pi^{+}$decay (and its charge conjugate) which has a branching ratio (BR) of about $3.8 \%$. The expected production yields $(\mathrm{BR} \times \mathrm{d} N / \mathrm{d} y$ at $y=0)$ for $\mathrm{D}^{0}$ (and $\left.\overline{\mathrm{D}^{0}}\right)$ mesons decaying in a $\mathrm{K}^{\mp} \pi^{ \pm}$pair in central $\mathrm{Pb}-$ $\mathrm{Pb}\left(0-5 \% \sigma^{\text {inel }}\right)$ at $\sqrt{s_{\mathrm{NN}}}=5.5 \mathrm{TeV}$, in minimum-bias $\mathrm{p}-\mathrm{Pb}$ collisions at $\sqrt{s_{\mathrm{NN}}}=8.8 \mathrm{TeV}$ and in pp collisions at $\sqrt{s}=14 \mathrm{TeV}$ are, in the order, $5.3 \times 10^{-1}, 3.7 \times 10^{-3}$ and $7.5 \times 10^{-4}$ per event.

Figure 1 (left) shows a sketch of the decay: the main feature of this topology is the presence of two tracks with

\footnotetext{
3 This value of the multiplicity can be taken as a conservative assumption, since extrapolations based on RHIC data predict $\mathrm{d} N_{\mathrm{ch}} / \mathrm{d} y=2000-3000$.
}
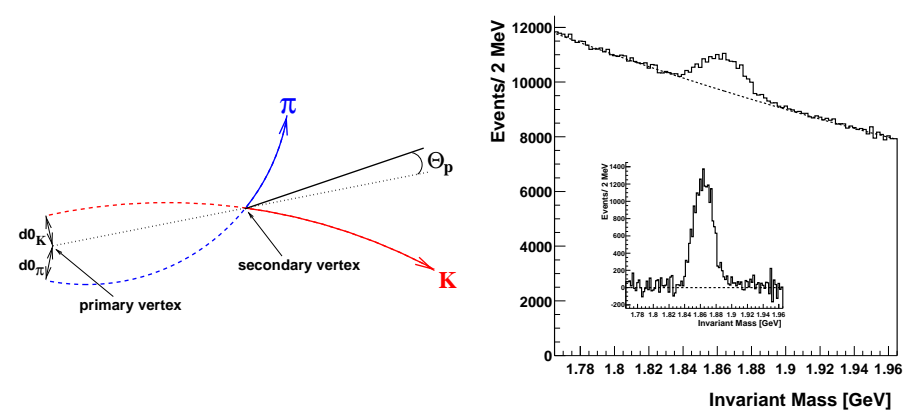

Fig. 1. Schematic representation of the $\mathrm{D}^{0} \rightarrow \mathrm{K}^{-} \pi^{+}$decay (left). $\mathrm{K} \pi$ invariant-mass distribution corresponding to $10^{7}$ central $\mathrm{Pb}-\mathrm{Pb}$ events (right); the background-subtracted distribution is shown in the insert. 


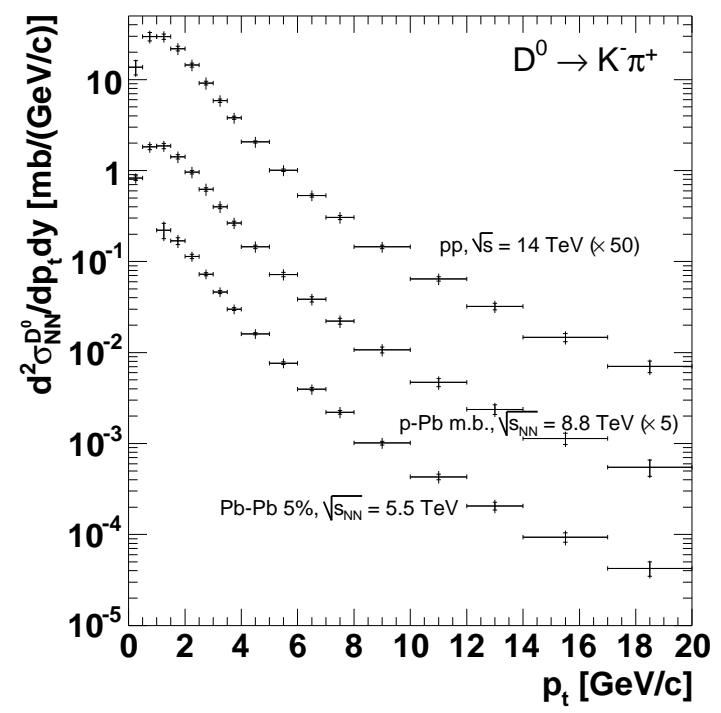

Fig. 2. $p_{\mathrm{t}}$-differential cross section per $\mathrm{NN}$ collision for $\mathrm{D}^{0}$ production, as expected to be measured with $10^{7}$ central $\mathrm{Pb}-\mathrm{Pb}$ events $10^{8}$ minimum-bias $\mathrm{p}-\mathrm{Pb}$ events, and $10^{9} \mathrm{pp}$ minimum-bias events. Statistical (inner bars) and quadratic sum of statistical and $p_{\mathrm{t}}$-dependent systematic errors (outer bars) are shown. A normalization error of $9 \%$ for $\mathrm{Pb}-\mathrm{Pb}, 9 \%$ for $\mathrm{p}-\mathrm{Pb}$ and $5 \%$ for $\mathrm{pp}$ is not shown.

impact parameters $d_{0} \sim 100 \mu \mathrm{m}$. The detection strategy 14 to cope with the large combinatorial background from the underlying event is based on:

1. selection of displaced-vertex topologies, i.e. two tracks with large impact parameters and small pointing angle $\Theta_{\mathrm{p}}$ between the $\mathrm{D}^{0}$ momentum and flight-line (see sketch in Fig. 11);

2. identification of the $\mathrm{K}$ track in the TOF detector;

3. invariant-mass analysis (see $p_{\mathrm{t}}$-integrated distribution in $\mathrm{Pb}-\mathrm{Pb}$ after selections in Fig. 11).

This strategy was optimized separately for $\mathrm{pp}, \mathrm{p}-\mathrm{Pb}$ and $\mathrm{Pb}-\mathrm{Pb}$ collisions, as a function of the $\mathrm{D}^{0}$ transverse momentum 15,13. As shown in Fig. 2 the accessible $p_{\mathrm{t}}$ range is $1-20 \mathrm{GeV} / c$ for $\mathrm{Pb}-\mathrm{Pb}$ and $0.5-20 \mathrm{GeV} / c$ for $\mathrm{pp}$ and $\mathrm{p}-$ $\mathrm{Pb}$, with a statistical error better than $15-20 \%$ and a systematic error (acceptance and efficiency corrections, centrality selection for $\mathrm{Pb}-\mathrm{Pb}$ ) better than $20 \%$. More details are given in Ref. 15,13.

For the case of pp collisions, the experimental errors on the $p_{\mathrm{t}}$-differential cross section are expected to be significantly smaller than the current theoretical uncertainty band from NLO pQCD calculations (estimated by varying the values of the charm quark mass and of the factorization and renormalization scales). The resulting 'data/theory plot in Fig. 3 shows that this will allow us to perform a sensitive test of the pQCD predictions for charm production at LHC energy.

We studied 16 the sensitivity for a comparison of the energy loss of charm quarks and of massless partons by considering:

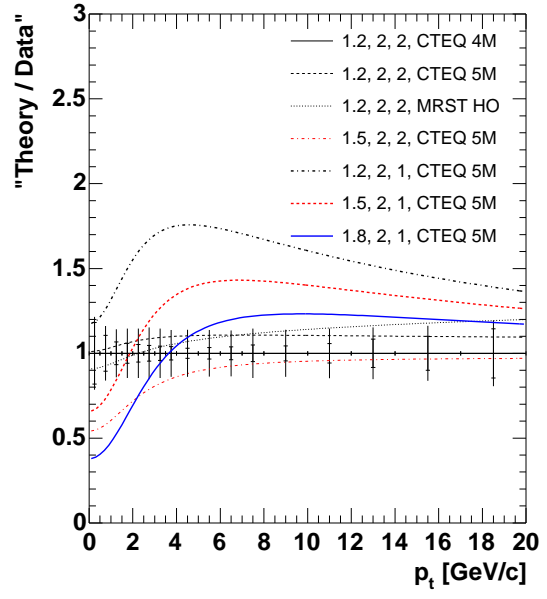

Fig. 3. Sensitivity on $\mathrm{d}^{2} \sigma^{\mathrm{D}^{0}} / \mathrm{d} p_{\mathrm{t}} \mathrm{d} y$, in pp at $14 \mathrm{TeV}$, compared to pQCD predictions obtained with different sets of input parameters: $m_{\mathrm{c}}[\mathrm{GeV}]$, the factorization and renormalization scales, in units of $m_{\mathrm{t}, \mathrm{c}}$, and the PDF set. The comparison is shown as a 'data/theory' plot. Error bars are defined as in Fig. 2

- the nuclear modification factor of D mesons as a function of $p_{\mathrm{t}}$

$$
R_{\mathrm{AA}}^{\mathrm{D}}\left(p_{\mathrm{t}}\right) \equiv \frac{1}{\left\langle N_{\text {coll }}\right\rangle} \times \frac{\mathrm{d} N_{\mathrm{AA}}^{\mathrm{D}} / \mathrm{d} p_{\mathrm{t}}}{\mathrm{d} N_{\mathrm{pp}}^{\mathrm{D}} / \mathrm{d} p_{\mathrm{t}}},
$$

which is used to characterize the medium-induced high$p_{\mathrm{t}}$ suppression - in central $\mathrm{Au}-\mathrm{Au}$ collisions at $\mathrm{RHIC}$, $R_{\mathrm{AA}}$ is found to be $\simeq 0.2$ for both $\pi^{0}$ and charged hadrons for $p_{\mathrm{t}}>4 \mathrm{GeV} / c[6$;

- the heavy-to-light ratio of the nuclear modification factors of D mesons and of charged hadrons:

$$
R_{\mathrm{D} / h}\left(p_{\mathrm{t}}\right) \equiv R_{\mathrm{AA}}^{\mathrm{D}}\left(p_{\mathrm{t}}\right) / R_{\mathrm{AA}}^{h}\left(p_{\mathrm{t}}\right)
$$

In Fig. 4 we compare our estimated sensitivity on $R_{\mathrm{AA}}^{\mathrm{D}}$ and $R_{\mathrm{D} / h}$ to theoretical calculation results [10] that implement radiative parton energy loss with medium density described by transport coefficient values in the range, $\hat{q}=25-100 \mathrm{GeV}^{2} / \mathrm{fm}$, expected for central $\mathrm{Pb}-\mathrm{Pb}$ collisions at $\sqrt{s_{\mathrm{NN}}}=5.5 \mathrm{TeV}$ on the basis of quenching measurements at RHIC. The experimental uncertainties, reported in Fig. 4 for the case $\hat{q}=50 \mathrm{GeV}^{2} / \mathrm{fm}$ and $m_{\mathrm{c}}=1.2 \mathrm{GeV}$, are discussed in detail in Refs. 16.15. The effect of nuclear shadowing, introduced via the EKS98 parameterization [4, is clearly visible in the $R_{\mathrm{AA}}$ without energy loss for $p_{\mathrm{t}} \lesssim 7 \mathrm{GeV} / c$. Above this region, only parton energy loss is expected to affect the nuclear modification factor of $\mathrm{D}$ mesons. The small difference between the theoretical $R_{\mathrm{AA}}$ predictions for $m_{\mathrm{c}}=0$ and $1.2 \mathrm{GeV}$ indicates that the charm quark behaves similarly to a light quark, as far as energy loss is concerned. Therefore, the enhancement of the heavy-to-light ratio $R_{\mathrm{D} / h}$ is a sensitive measurement, free of mass effects, to study the colour-charge dependence of parton energy. As shown by the error bars in the figure, $R_{\mathrm{D} / h}$ can be measured with good accuracy 

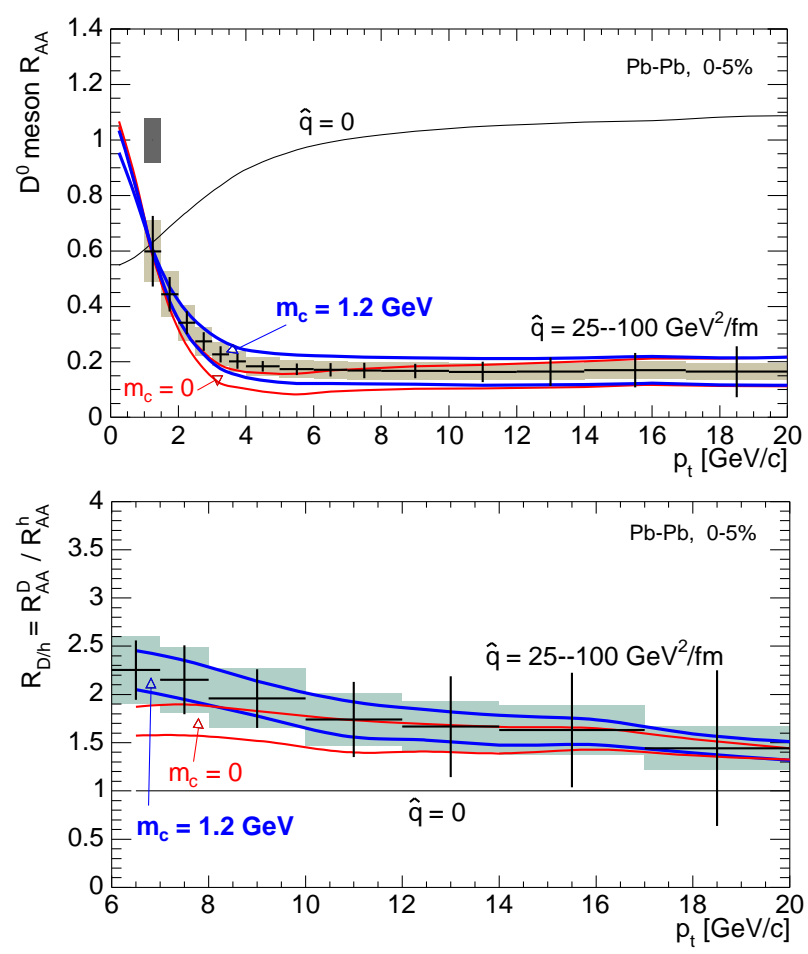

Fig. 4. Nuclear modification factor for $D^{0}$ mesons (top) and heavy-to-light ratio of the nuclear modification factors for $\mathrm{D}^{0}$ mesons and for charged hadrons (bottom). Predictions [10] with and without the effect of the charm mass are shown for the medium density range $\hat{q}=25-100 \mathrm{GeV}^{2} / \mathrm{fm}$. Errors corresponding to the case ' $\hat{q}=50 \mathrm{GeV}^{2} / \mathrm{fm}$ and $m_{\mathrm{c}}=1.2 \mathrm{GeV}$ ' are reported: bars $=$ statistical, shaded area $=$ systematic.

(as it is a double ratio $(\mathrm{AA} / \mathrm{pp}) /(\mathrm{AA} / \mathrm{pp})$, some common systematic uncertainties cancel out).

\section{Measurement of beauty production in the semi-electronic decay channel}

The production of open beauty can be studied by detecting the semi-electronic decays of beauty hadrons, mostly B mesons. Such decays have a branching ratio of $\simeq 10 \%$ (plus $10 \%$ from cascade decays $\mathrm{b} \rightarrow \mathrm{c} \rightarrow e$, that only populate the low- $p_{\mathrm{t}}$ region in the electron spectrum). The expected yields $(\mathrm{BR} \times \mathrm{d} N / \mathrm{d} y$ at $y=0)$ for $\mathrm{b} \rightarrow e+X$ plus $\mathrm{b} \rightarrow \mathrm{c}(\rightarrow e+X)+X^{\prime}$ in central $\mathrm{Pb}-\mathrm{Pb}\left(0-5 \% \sigma^{\text {inel }}\right)$ at $\sqrt{s_{\mathrm{NN}}}=5.5 \mathrm{TeV}$ and in in pp collisions at $\sqrt{s}=14 \mathrm{TeV}$ are $1.8 \times 10^{-1}$ and $2.8 \times 10^{-4}$ per event, respectively.

The main sources of background electrons are: (a) decays of D mesons; (b) neutral pion Dalitz decays $\pi^{0} \rightarrow$ $\gamma e^{+} e^{-}$and decays of light mesons (e.g. $\rho$ and $\omega$ ); (c) conversions of photons in the beam pipe or in the inner detector layers and (d) pions misidentified as electrons. Given that electrons from beauty have average impact parameter $d_{0} \simeq 500 \mu \mathrm{m}$ and a hard momentum spectrum, it is possible to obtain a high-purity sample with a strategy that relies on:

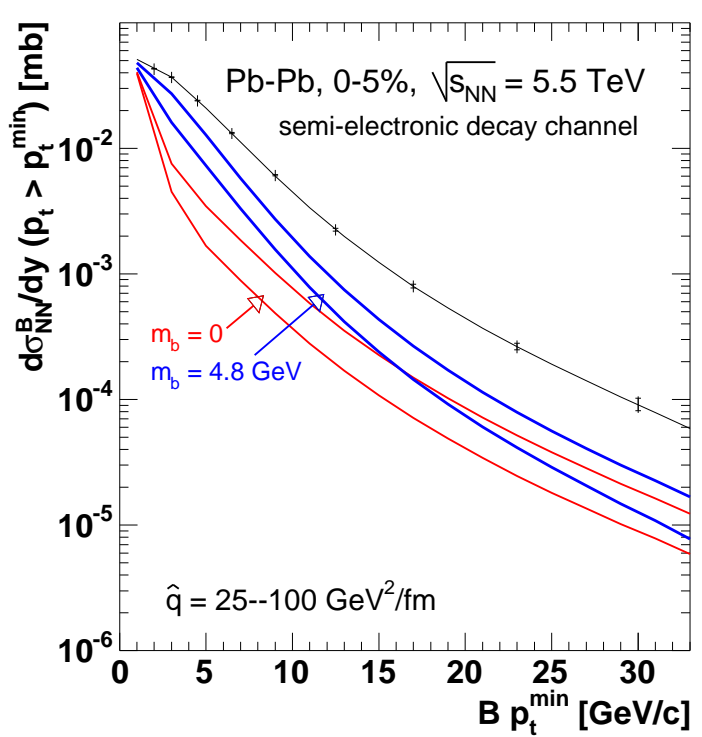

Fig. 5. Minimum- $p_{\mathrm{t}}$-differential production cross section per $\mathrm{NN}$ collision for B mesons at $y=0$, as expected to be measured from semi-electronic decays with $10^{7}$ central $\mathrm{Pb}-\mathrm{Pb}$ events. Statistical errors (inner bars) and quadratic sum of statistical and $p_{\mathrm{t}}$-dependent systematic errors (outer bars) are shown. A normalization error of $9 \%$ is not shown. Suppression predictions [10 with and without the effect of the beauty mass are shown for the medium density range $\hat{q}=25-100 \mathrm{GeV}^{2} / \mathrm{fm}$.

1. electron identification with a combined $\mathrm{d} E / \mathrm{d} x$ (TPC) and transition radiation selection, which is expected to reduce the pion contamination by a factor $10^{4}$;

2. impact parameter cut to reject misidentified $\pi^{ \pm}$and $e^{ \pm}$from Dalitz decays and $\gamma$ conversions (the latter have small impact parameter for $p_{\mathrm{t}} \gtrsim 1 \mathrm{GeV} / c$ );

$3 . p_{\mathrm{t}}$ cut to reject electrons from charm decays.

As an example, with $d_{0}>200 \mu \mathrm{m}$ and $p_{\mathrm{t}}>2 \mathrm{GeV} / c$, the expected statistics of electrons from $\mathrm{b}$ decays is $8 \times 10^{4}$ for $10^{7}$ central $\mathrm{Pb}-\mathrm{Pb}$ events, allowing the measurement of electron-level $p_{\mathrm{t}}$-differential cross section in the range $2<$ $p_{\mathrm{t}}<18 \mathrm{GeV} / c$. The residual contamination of about $10 \%$, accumulated in the low- $p_{\mathrm{t}}$ region, of electrons from prompt charm decays, from misidentified charged pions and $\gamma$ conversion electrons can be evaluated and subtracted using a Monte Carlo simulation tuned to reproduce the measured cross sections for pions and $\mathrm{D}^{0}$ mesons. A MonteCarlo-based procedure can then be used to compute, from the electron-level cross section, the B-level cross section $\mathrm{d} \sigma^{\mathrm{B}}\left(p_{\mathrm{t}}>p_{\mathrm{t}}^{\mathrm{min}}\right) / \mathrm{d} y[13$. In Fig. [5] we show this cross section for central $\mathrm{Pb}-\mathrm{Pb}$ collisions with the estimated statistical and systematic uncertainties. The covered range is $2<p_{\mathrm{t}}^{\min }<30 \mathrm{GeV} / c$.

The predicted suppression of the B meson $p_{\mathrm{t}}^{\mathrm{min}}$-differential cross section due to b quark energy loss is also plotted in Fig.5 The transport coefficient range $25-100 \mathrm{GeV}^{2} / \mathrm{fm}$ is considered and the two bands represent the results for $m_{\mathrm{b}}=0$ and $4.8 \mathrm{GeV}$; the two bands are well separated up to $p_{\mathrm{t}}^{\min } \simeq 15 \mathrm{GeV} / c$. The quenching predictions are shown only for illustration, since the study of the B me- 
son suppression will have to be performed by using as a reference the cross section measured in pp collisions. The sensitivity of this study is currently being investigated.

\section{Measurement of beauty production in the semi-muonic decay channel}

Beauty production can be measured also in the ALICE forward muon spectrometer, $-4<\eta<-2.5$, analyzing the single-muon $p_{\mathrm{t}}$ distribution and the opposite-sign dimuons invariant mass distribution [17,13.

The main backgrounds to the 'beauty muon' signal are $\pi^{ \pm}, \mathrm{K}^{ \pm}$and charm decays. The cut $p_{\mathrm{t}}>1.5 \mathrm{GeV} / c$ is applied to all reconstructed muons in order to increase the signal-to-background ratio. For the opposite-sign dimuons, the residual combinatorial background is subtracted using the technique of event-mixing and the resulting distribution is subdivided into two samples: the low-mass region, $M_{\mu^{+} \mu^{-}}<5 \mathrm{GeV}$, dominated by di-muons originating from a single $\mathrm{b}$ quark decay through $\mathrm{b} \rightarrow \mathrm{c}(\rightarrow$ $\left.\mu^{+}\right) \mu^{-}\left(\mathrm{BD}_{\text {same }}\right)$, and the high-mass region, $5<M_{\mu^{+} \mu^{-}}<$ $20 \mathrm{GeV}$, dominated by $\mathrm{b} \overline{\mathrm{b}} \rightarrow \mu^{-} \mu^{+}$, with each muon coming from a different quark in the pair $\left(\mathrm{BB}_{\text {diff }}\right)$. Both samples have a background from $c \overline{\mathrm{c}} \rightarrow \mu^{+} \mu^{-}$and a fit is performed to extract the charm- and beauty-component yields. The single-muon $p_{\mathrm{t}}$ distribution has three components with different slopes: $\mathrm{K}$ and $\pi$, charm, and beauty decays. The first component is subtracted on the basis of the identified hadron spectra measured in the central barrel. Then, a fit technique allows to extract a $p_{\mathrm{t}}$ distribution of muons from beauty decays. A Monte Carlo procedure, similar to that used for semi-electronic decays, allows to extract B-level cross sections for the data sets (low-mass $\mu^{+} \mu^{-}$, high-mass $\mu^{+} \mu^{-}$, and $p_{\mathrm{t}}$-binned singlemuon distribution), each set covering a different B-meson $p_{\mathrm{t}}>p_{\mathrm{t}}^{\min }$ region. The results using only the single muons are shown in Fig. 6] Since only minimal cuts are applied, the reported statistical errors (inner bars) are very small and the high- $p_{\mathrm{t}}$ reach is excellent. The main sources of systematic errors (outer bars) are: corrections for acceptance and efficiency, subtraction of the background muons from charged pion and kaon decays, and fit procedure to separate the beauty and charm components.

\section{Conclusions}

Heavy quarks, abundantly produced at LHC energies, will allow to address several physics issues, in pp, pA and AA collisions. In particular, they provide tools to: probe, via parton energy loss and its predicted colour-charge and mass dependences, the dense medium formed in $\mathrm{Pb}-\mathrm{Pb}$ collisions; probe, in pp collisions, the pQCD calculations parameters space; probe the small- $x$ regime of the PDFs, where saturation effects are expected to be important.

The excellent tracking, vertexing and particle identification performance of ALICE will allow to fully explore this rich phenomenology, as we have shown with some specific examples on D and B meson measurements.

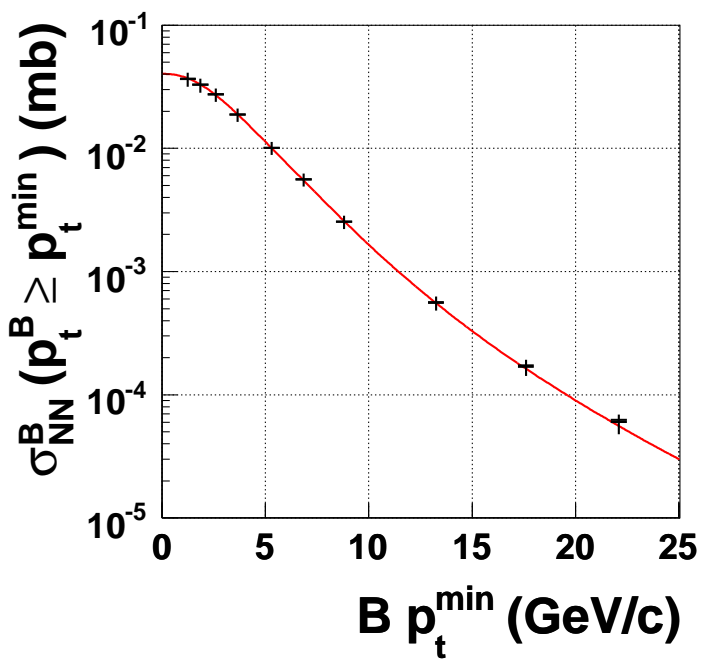

Fig. 6. Minimum- $p_{\mathrm{t}}$-differential production cross section per $\mathrm{NN}$ collision for $\mathrm{B}$ mesons with $-4<y<-2.5$ in central $\mathrm{Pb}-\mathrm{Pb}$ collisions, as expected to be measured from the singlemuon data set. Statistical errors (inner bars) corresponding to $4 \times 10^{8}$ events and $p_{\mathrm{t}}$-dependent systematic errors (outer bars) are shown. A normalization error of $10 \%$ is not shown.

\section{References}

1. ALICE Collaboration, Physics Performance Report Vol. I, J. Phys. G 30, 1517 (2003) (CERN/LHCC 2003049).

2. M. Mangano, P. Nason and G. Ridolfi, Nucl. Phys. B 373, 295 (1992).

3. N. Carrer and A. Dainese, ALICE Internal Note, ALICEINT-2003-019 (2003), arXiv:hep-ph/0311225

4. K.J. Eskola, V.J. Kolhinen, C.A. Salgado, Eur. Phys. J. C 9, 61 (1999).

5. A. Dainese, R. Vogt, M. Bondila, K.J. Eskola and V.J. Kolhinen, J. Phys. G 30, 1787 (2004).

6. T. Peitzmann, these proceedings.

7. Yu.L. Dokshitzer and D.E. Kharzeev, Phys. Lett. B 519, 199 (2001).

8. N. Armesto, C.A. Salgado and U.A. Wiedemann, Phys. Rev. D 69, 114003 (2004).

9. M. Djordjevic and M. Gyulassy, Phys. Lett. B 560, 37 (2003); Nucl. Phys. A 733, 265 (2004).

10. N. Armesto, A. Dainese, C.A. Salgado and U.A. Wiedemann, Phys. Rev. D 71, 054027 (2005).

11. H.-A. Gustafsson, these proceedings.

12. C. Adler, these proceedings.

13. ALICE Collaboration, Physics Performance Report Vol. II, in preparation.

14. N. Carrer, A. Dainese and R. Turrisi, J. Phys. G 29, 575 (2003).

15. A. Dainese, Ph.D. Thesis (2003), arXiv:nucl-ex/0311004

16. A. Dainese, Eur. Phys. J. C 33, 495 (2004).

17. R. Guernane et al., ALICE Internal Note, ALICE-INT2005-018 (2005). 
